2

\title{
COHOMOLOGY GAPS FOR SHEAVES ON THREEFOLDS
}

\author{
EDOARDO BALLICO AND ELIZABETH GASPARIM
}

Communicated by Vassil V. Tsanov

\begin{abstract}
We discuss cohomological invariants of framed reflexive sheaves on $(n \geq 3)$-folds and show that some numerically admissible values of cohomology do not occur.
\end{abstract}

\section{Introduction}

We first mention briefly our physics motivation. The paper [3] showed gaps on the values of topological charges for instantons on local surfaces. Using the KobayashiHitchin $(\mathrm{KH})$ correspondence (see [6] for the general theory on KH correspondence) this result is equivalent to the existence of gaps on the value of the holomorphic Euler characteristic of framed sheaves over local surfaces ([3], Lemma 6.5 and Proposition 6.7). For ( $n \geq 3)$-folds there is no analogue of the beautiful KH correspondence, simply because the equation defining anti-self-dual connections only makes sense in four dimensions. Nevertheless, sheaves on threefolds are very interesting for questions in string theory and appear in various physical theories that can be regarded as higher dimensional analogues of instanton counting, such as counting of dyons and Bogomolnyi-Pasad-Sommerfeld (BPS) states. In further generality sheaves on threefolds occur as D-branes in string theory and cohomological values of such sheaves can be interpreted as some measure of energy within the charge of a brane. The authors of this paper are mathematicians, and do not aim here to propose a definition of brane charge. We have encountered many different definitions in the literature. Nevertheless, there is a common feature that by and large such definitions involve cohomological invariants of sheaves on threefolds. Thus, we expect that gaps on cohomological invariants of such sheaves shall have interesting physics interpretations. We choose to present this paper in a physics oriented journal in the hope that our result may inspire researchers to find a physical counterpart of the mathematical phenomenon we observed. On the other hand, cohomological invariants of bundles and sheaves are of interest in geometry in their own right and have applications to fundamental questions in geometry, for instance 
they appear as obstructions to existence of metrics with prescribed curvature, see Chapter 6 of [2]. Hence cohomological gaps lie behind vanishing of obstructions.

The main theorem we present here shows that if $E$ is some reflexive sheaf of rank $r \geq 2$ on a smooth projective threefold $X$ framed over a smooth, ample, and effective divisor, then $h^{0}(X, E) \neq r-1$. The summary goes as follows. In this paper we show existence of gaps on cohomology values for sheaves on $(n \geq 3)$-folds and study their filtrability properties (Theorem 11). We also discuss the deformation functor (Definition 3 ) of framed sheaves on threefolds and present a cohomological criterion for rigidity (Theorem 12). We present several concrete examples focusing on ruled and doubly-ruled threefolds (Sections 2 and 5).

\section{Doubly-ruled Threefolds}

In this section we define doubly-ruled threefolds, which will illustrate our theorems, and we set up the terminology of splitting types.

Fix a smooth and connected projective curve $C$ of genus $q \geq 0$ and a rank twovector bundle $G$ on $C$. Set $S:=\mathbb{P}(G)$. Let $u: S \rightarrow C$ denote the ruling of $S$. Fix a rank two-vector bundle $A$ on $S$ and set $X:=\mathbb{P}(A)$. Let $v: X \rightarrow S$ be a ruling and $D \subset X$ a section of $v$ such that $\mathcal{O}_{X}(D)$ is ample. Hence, we have two rulings and a section

$$
X \underset{v}{\stackrel{D}{\longrightarrow}} S \underset{u}{\longrightarrow} C
$$

such that $L:=\mathcal{O}_{D}(D)$ is an ample line bundle on $D$. By assumption $v \mid D \rightarrow S$ is an isomorphism and we will use it to see $u: D \rightarrow C$ as a ruling of $D$. Here

$$
h^{1}\left(X, \mathcal{O}_{X}\right)=h^{1}\left(D, \mathcal{O}_{D}\right)=q
$$

Definition 1. Set $a:=u^{-1}(p) \cdot L$ for any $p \in C$. The integer $a$ is called the fiber degree of $L$.

Fix a rank $r$ vector bundle $E_{0}$ on $D$. For any $p \in C$ let $a_{1, p} \geq \cdots \geq a_{r, p}$ be the splitting type of $E_{0} \mid u^{-1}(p)$.

Definition 2. Set $a_{i}:=a_{i, p}$, where $p$ is a general point of $C$. The set of integers $a_{1} \geq \cdots \geq a_{r}$ are called the generic splitting type of $E_{0}$. 
Thus we have

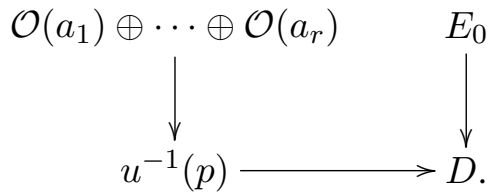

Let $b_{1} \geq \cdots \geq b_{r^{2}}$ denote the generic splitting type of $\operatorname{End}\left(E_{0}\right)$. Notice that $b_{r^{2}}=a_{r}-a_{1}, b_{1}=a_{1}-a_{r}$, and $b_{1}-b_{r^{2}}=2\left(a_{1}-a_{r}\right)$.

\section{Deformations of Framed Bundles}

Let $X$ be a smooth and connected projective $n$-fold and $D$ a divisor on $X$. Fix a vector bundle $E$ on $X$ and consider the exact sequence

$$
0 \rightarrow \operatorname{End}(E)(-D) \rightarrow \operatorname{End}(E) \rightarrow \operatorname{End}(E \mid D) \rightarrow 0
$$

The cohomology exact sequence of (2) induces a linear map

$$
\rho: H^{1}(X, \operatorname{End}(E)) \rightarrow H^{1}(D, \operatorname{End}(E \mid D))
$$

and a surjection

$$
\eta: H^{1}(X, \operatorname{End}(E)(-D)) \rightarrow \operatorname{Ker}(\rho)
$$

Definition 3. $\operatorname{Ker}(\rho)$ is the tangent space to the deformation functor $\Psi_{E_{0}}$ of $E$ with prescribed restriction $E_{0}:=E \mid D$ to $E$.

Let $X$ be a doubly ruled threefold as in (1). Fix any vector bundle $E_{0}$ on $D$ and use the isomorphism $v \mid D$ to identify $E_{0}$ with a vector bundle $F$ on $S$. Set $\mathbf{E}_{\mathbf{0}}:=v^{*}(F)$. Then $\mathbf{E}_{\mathbf{0}}$ is a vector bundle on $X$ such that $\mathbf{E}_{\mathbf{0}} \mid D \cong E_{0}$.

Definition 4. We will say that $\mathbf{E}_{\mathbf{0}}$ is the trivial extension of $E_{0}$ to $X$.

We first give a couple of examples of the extremal cases: i) when $\mathbf{E}_{\mathbf{0}}$ does not have any non-trivial deformation with fixed restriction to $D$, and ii) when the map $\eta$ appearing in (3) is an isomorphism.

Example 5. Take $E_{0} \cong \mathcal{O}_{D}^{\oplus r}$. Hence $\mathbf{E}_{\mathbf{0}} \cong \mathcal{O}_{\mathbf{X}}^{\oplus \mathbf{r}}$. Kodaira's vanishing gives $h^{1}(X, \operatorname{End}(E)(-D))=0$. Hence, a trivial vector bundle is rigid with respect to the deformations which preserve its restriction to $D$. More generally, for $i=0,1$ and for all integers $k>0$ we have $h^{i}\left(D, \operatorname{End}\left(E_{0}\right) \otimes L^{* \otimes k}\right)=0$, again by Kodaira vanishing. Since $h^{1}\left(X, \mathcal{O}_{X}\right)=q$, a trivial vector bundle on $X$ is rigid without any condition on its restriction to $D$ if and only if $q=0$. 
Example 6. Consider the case when $E_{0}$ is simple and $L$ is effective. Because $h^{0}(D, L)>0$ and $L \neq \mathcal{O}_{D}$, we obtain that $h^{0}\left(D, \operatorname{End}\left(E_{0}\right) \otimes L^{* \otimes k}\right)=0$ for all integers $k>0$ and the corresponding maps $\eta$ of (3) are isomorphisms.

Example 7. Assume that the fiber degree is related to the splitting type so that $a>a_{1}-a_{r}$ (see Definitions 1 and 2$)$. Restrict $\operatorname{End}\left(E_{0}\right)$ to a general fiber $T$ of the ruling $u: D \rightarrow C$. Notice that $\operatorname{End}\left(E_{0}\right) \otimes L^{* \otimes k} \mid T$ has splitting type $b_{1}-k a \geq$ $\cdots \geq b_{r}-k a$. Since for all $k>0$ we have $b_{1}=a_{1}-a_{r}, b_{1}-k a<0$, then $h^{0}\left(D, \operatorname{End}\left(E_{0}\right) \otimes L^{* \otimes k}\right)=0$ for all integers $k>0$, and the corresponding maps $\eta$ of (3) are isomorphisms.

Of course the above examples are special situations, and in general there will be deformations of $E_{0}$ and the map $\eta$ will fail to be an isomorphism. We can also regard this situation from the opposite viewpoint but instead of looking at $\mathbf{E}_{\mathbf{0}}$ as an extension of $E_{0}$, we can regard $\mathbf{E}_{\mathbf{0}}$ as a framed bundle, that is, $\mathbf{E}_{\mathbf{0}}$ is given together with its restriction to a divisor. We then find that such framing imposes restrictions to the cohomology of $\mathbf{E}_{\mathbf{0}}$.

\section{Cohomology of Framed Bundles}

Proposition 8. Let $X$ be a smooth and connected projective $n$-fold, $n \geq 3$, and $D$ an effective, smooth and ample divisor of $X$. Let $E$ be a rank $r$ vector bundle on $X$ such that $E \mid D$ is trivial. Then $E$ is trivial.

Proof: For any integer $t$ consider the exact sequence

$$
0 \rightarrow E(-(t+1) D) \rightarrow E(-t D) \rightarrow E(-t D) \mid D \rightarrow 0 .
$$

Since $\mathcal{O}_{D}(D)$ is ample, $E \mid D$ is trivial and $\operatorname{dim}(D) \geq 2$, by Kodaira vanishing we have $h^{i}(D, E(-t D) \mid D)=0$ for $i=0,1$ and all $t \geq 1$. Since $E$ has depth at least two at each point of $X$ a theorem of Serre gives $h^{i}(X, E(-t D))=0$ for $i=0,1$ and $t \gg 0$. Hence, by decreasing induction on $t$ and using (4) we get $h^{i}(X, E(-t D))=0$ for $i=0,1$, and all $t \geq 1$. Thus, sequence (4) gives

$$
h^{0}(X, E)=h^{0}(D, E \mid D) .
$$

Since $E \mid D$ is trivial, we get $h^{0}(X, E)=r$. Let

$$
e_{E}: H^{0}(X, E) \otimes \mathcal{O}_{X} \rightarrow E
$$

denote the evaluation map. Since $e_{E}$ is a map between vector bundles of the same rank, it drops rank either at no point of $X$, or at all points of $X$, or else along a 
non-empty hypersurface of $X$. The exact sequence (4) for $t=1$ together with (5) show that the restriction map $H^{0}(X, E) \rightarrow H^{0}(D, E \mid D)$ is an isomorphism. Since $E \mid D$ is trivial, $e_{E}$ does not drop rank at any point of $D$. The ampleness of $D$ implies that $D$ intersects every curve contained in $X$ and hence every surface contained in $X$. Hence $e_{E}$ drops rank at no point of $X$, and consequently is an isomorphism.

Remark 9. (choice of framing) Let $X$ be a smooth and connected projective $n$ fold, $n \geq 3$, and $D$ an effective and ample divisor of $X$. Fix $R \in \operatorname{Pic}(X)$ and $a$ rank $r$ vector bundle $G$ on $D$. There is a natural bijection $E \mapsto E \otimes R$ between the rank $r$ vector bundles (or reflexive sheaves) $E$ on $X$ such that $E \mid D \cong G$ and the rank $r$ vector bundles (resp. reflexive sheaves) $F$ on $X$ such that $F \mid D \cong$ $G \otimes(R \mid D)$. Hence we see that we gain nothing if instead of assuming $E \mid D \cong \mathcal{O}_{D}^{\oplus r}$ we assume $E \mid D \cong(R \mid D)^{\oplus r}$. To get a more interesting set-up we must either weaken the assumption "D-ample" or else take a different vector bundle on $D$ instead of a trivial vector bundle as a framing.

Proposition 10. Let $X$ be a smooth and connected projective $n$-fold, $n \geq 3$, and $D$ an effective, smooth and ample divisor of $X$. Let $E$ be a rank $r \geq 2$ reflexive sheaf on $X$ such that $E \mid D \cong \mathcal{O}_{D}^{\oplus r}$. Then $\operatorname{det}(E) \cong \mathcal{O}_{X}$, and $h^{0}(X, E) \leq r$ with equality $h^{0}(X, E)=r$ if and only if $E$ is trivial.

Proof: We follow similar lines as in the proof of Proposition 8. By assumption $E$ is locally free in a neighborhood of $D$ and $\operatorname{det}(E)$ is a reflexive rank one sheaf. Since $X$ is smooth, $\operatorname{det}(E)$ is a line bundle ([5], Proposition 1.9). Since $D$ is ample and $\operatorname{det}(E) \mid D \cong \operatorname{det}(E \mid D) \cong \mathcal{O}_{D}$, we get $\operatorname{det}(E) \cong \mathcal{O}_{X}$. Hence, we have the exact sequence (4) in this case as well. To get the inequality $h^{0}(X, E) \leq r$ we just use that $E$ is torsion free thus with depth $>0$ at each point of $X$ and this gives $h^{0}(X, E(-t D))=0$ for $t \gg 0$ by a cohomological property of depth (Serre). If $h^{0}(X, E)=r$, then the evaluation map $e_{E}: H^{0}(X, E) \otimes \mathcal{O}_{X} \rightarrow E$ is surjective. Given that $e_{E} \mid D$ is an isomorphism and $D$ is ample, $e_{E}$ is an isomorphism outside a codimension two algebraic subset of $X$. Since $E$ is reflexive, $e_{E}$ is an isomorphism ([5], Proposition 1.6).

Theorem 11. Let $X$ be a smooth and connected projective $n$-fold with $n \geq 3$, and $D$ an effective, smooth and ample divisor of $X$. Let $E$ be a reflexive sheaf on $X$ of rank $r \geq 2$ such that $E \mid D \cong \mathcal{O}_{D}^{\oplus r}$ and $v:=h^{0}(X, E)>0$. We have

- (gap on cohomology) $v \neq r-1$. 
- (filtrability) If $v \neq r$, then there exists a rank $r-v$ reflexive sheaf $G$ on $X$ such that $h^{0}(X, G)=0, G \mid D \cong \mathcal{O}_{D}^{\oplus(r-v)}$, and $E$ fits into an exact sequence

$$
0 \rightarrow \mathcal{O}_{X}^{\oplus v} \rightarrow E \rightarrow G \rightarrow 0
$$

Proof: Proposition 10 gives $v \leq r$. Assume $v \leq r-1$ and consider the evaluation map $e_{E}$ of (6). In a neighborhood of $D$ the cokernel $G:=\operatorname{Coker}\left(e_{E}\right)$ is a trivial rank $r-v$ vector bundle. Since $D$ is ample, we get that $G$ is locally free outside finitely many points of $X$. Fix any $P \in X$ at which $G$ is not locally free. The exact sequence

$$
0 \rightarrow H^{0}(X, E) \otimes \mathcal{O}_{X} \rightarrow E \rightarrow G \rightarrow 0
$$

shows that the cokernel $G$ has depth at least $\max \left\{\operatorname{depth}(G), \operatorname{depth}\left(\mathcal{O}_{X}\right)-1\right\}$ at $P$ ([1], Corollary 18.6). Hence $G$ has depth at least two at each point of $X$. Let $G^{\prime}$ be the torsion part of $G$. Assume $G^{\prime} \neq 0$. We saw that $G^{\prime}$ has finite support and depth at least two. However, any non-zero sheaf supported at finitely many points has depth 0 , a contradiction. Thus $G$ is torsion free, and consequently it is reflexive ([5], Proposition 1.3). Now assume $v=r-1$. Since $G$ is reflexive with rank one and $X$ is smooth, $G$ is a line bundle. Since $\operatorname{det}(G) \cong \mathcal{O}_{X}$ (Proposition 10), taking determinants in (9) we get $G \cong \mathcal{O}_{X}$ Thus $E$ is an extension of $\mathcal{O}_{X}$ by $\mathcal{O}_{X}^{(r-1)}$, say induced by the extension class $\epsilon \in \operatorname{Ext}^{1}\left(X, \mathcal{O}_{X}, \mathcal{O}_{X}^{(r-1)}\right) \cong H^{1}\left(X, \mathcal{O}_{X}^{(r-1)}\right)$. Since $D$ is ample, Kodaira's vanishing gives $h^{1}\left(X, \mathcal{O}_{X}(-D)\right)=0$. Hence the restriction map $u: H^{1}\left(X, \mathcal{O}_{X}^{(r-1)}\right) \rightarrow H^{1}\left(D, \mathcal{O}_{X}^{(r-1)}\right)$. Since $E \mid D \cong \mathcal{O}_{D}^{\oplus r}$, we have $u(\epsilon)=$ 0 . Since $u$ is injective, we get $E \cong \mathcal{O}_{X}^{\oplus r}$. Hence $v=r$, contradiction.

Now we consider vector bundles $E$ and $B$ on $X$ and $D$ resp. with $\left.E\right|_{D} \simeq B$ and we will give conditions on $B$ (we stress, on $B$, not on $E$ ) which assure that $E$ is, up to isomorphism, the only vector bundle on $X$ with $B$ as its restriction to $D$.

Theorem 12. (cohomological criterion for rigidity) Let $X$ be a smooth and connected projective $n$-fold, $n \geq 3$, and $D$ an effective, smooth and ample divisor of $X$. Fix a rank $r$ vector bundle $B$ on $D$ such that $h^{i}(D, \operatorname{Hom}(B, B) \otimes$ $\left.\mathcal{O}_{D}(-t D)\right)=0$ for $i=0,1$, and all integers $t \geq 1$. Then, up to isomorphisms, there is at most one vector bundle $E$ on $X$ such that $E \mid D \cong B$.

Proof: Let $E, A$ be vector bundles on $X$ such that $E|D \cong A| D \cong B$. For any integer $x$ there is an exact sequence on $X$

$$
\begin{aligned}
0 \rightarrow \operatorname{Hom}(E, A)(-(x+1) D) & \rightarrow \operatorname{Hom}(E, A)(-x D) \\
& \rightarrow \operatorname{Hom}(B, B) \otimes \mathcal{O}_{D}(-x D) \rightarrow 0 .
\end{aligned}
$$


Since $\operatorname{Hom}(E, A)$ is locally free, $X$ is smooth and $\operatorname{dim}(X) \geq 2$, a theorem of Serre gives $h^{i}(X, \operatorname{Hom}(E, A)(-z D))=0$ if $z \gg 0$. Hence, from (8) our assumptions easily give $h^{1}(X, \operatorname{Hom}(B, B)(-D))=0$. Thus (8) implies that the identity map $\operatorname{Id}_{B}: B \rightarrow B$ lifts to a homomorphism $u: E \rightarrow A$ which is an isomorphism at each point of $D$. Since $X$ is connected and $E, A$ are vector bundles on $X$ with the same rank, either $u$ is an isomorphism or it drops rank on an effective divisor $T$ (the zero locus of the determinant of $u$ ). Since $u \mid D=\operatorname{Id}_{B}$ is an isomorphism, $T \cap D=\emptyset$. But given that $D$ is ample, it meets every curve of $X$ and hence every non-empty effective divisor of $D$, a contradiction.

Remark 13. (nonexistence of extensions) Of course, for many vector bundles $B$ on $D$ there is no vector bundle extension to $X$ such that $E \mid D \cong B$ (take for instance the case $r=2, X=\mathbb{P}^{n}, n \geq 3$, and $D$ a hyperplane).

Example 14. Let $X$ be a smooth and connected projective $n$-fold, $n \geq 3$, and $D$ an effective, smooth and ample divisor of $X$. For any integer $x \geq 0$ let $D_{X}^{(x)}$ denote the infinitesimal neighborhood of order $x$ of $D$ in $X$, i.e., the closed subscheme of $X$ with $\mathcal{O}_{X}(-(x+1) D)$ as its ideal sheaf. For instance $D_{X}^{0}=D$. Fix any vector bundle $B$ on $D$. Since $D$ is ample divisor and $\operatorname{dim}(D)=n-1 \geq 2$, there exists an integer $t_{0}>0$ (depending only on $D, B$ and $\left.X\right)$ such that $h^{i}(D, \operatorname{Hom}(B, B) \otimes$ $\left.\mathcal{O}_{D}(-t D)\right)=0$ for $i=0,1$ and all integers $t \geq t_{0}$. Let $E, E^{\prime}$ be vector bundles on $X$ such that $E\left|D_{X}^{\left(t_{0}-1\right)} \cong E^{\prime}\right| D_{X}^{\left(t_{0}-1\right)}$ and $E\left|D \cong E^{\prime}\right| D \cong B$. We claim that $E \cong E^{\prime}$. Indeed, the proof of Proposition 12 shows that we may lift any isomorphism $\tau: E\left|D_{X}^{\left(t_{0}-1\right)} \rightarrow E^{\prime}\right| D_{D}^{\left(t_{0}-1\right)}$ to a morphism $u: E \rightarrow E^{\prime}$. Since $u|D=\tau| D$ is an isomorphism, $E$ and $E^{\prime}$ are vector bundles with the same rank and $D$ is ample, $u$ is an isomorphism (see the proof of Proposition 8).

Example 15. Take $X, D, B$ as in Example 14. Here we give an example where we may take $t_{0}=1$ and hence $B$ has at most one extension to $X$. Assume the existence of an increasing filtration $\left\{B_{i}\right\}_{0 \leq i \leq r}$ of $B$ by subsheaves such that $B_{0}=0$, $B_{r}=B$, and each $B_{i} / B_{i-1}, 1 \leq i \leq r$, is an algebraically trivial line bundle. Hence $\operatorname{Hom}(B, B)$ has an increasing filtration $\left\{A_{i}\right\}_{0 \leq i \leq r^{2}}$ by subsheaves such that $A_{0}=0, A_{r^{2}}=\operatorname{Hom}(B, B)$ and each $A_{i} / A_{i-1}, 1 \leq i \leq r^{2}$, is an algebraically trivial line bundle. Since ampleness is a numerical property for line bundles by the Nakai criterion of ampleness (see [4]), each $\left(A_{i} / A_{i-1}\right)^{*} \otimes \mathcal{O}_{D}(D)$, $1 \leq i \leq r^{2}$, is an ample line bundle on $D$. Using the short exact sequences of sheaves associated to the filtration $\left\{A_{i}\right\}_{0 \leq i \leq r^{2}}$ together with Kodaira vanishing, we obtain $h^{i}\left(D, \operatorname{Hom}(B, B) \otimes \mathcal{O}_{D}(-t D)\right)=0$ for $i=0,1$ and all integers $t>0$. Thus, we can apply Theorem 12 to find a unique extension of $B$. If $q:=h^{1}\left(D, \mathcal{O}_{D}\right)>0$, then the set of all algebraically trivial line bundles on $D$ 
is an Abelian variety of dimension $q$ and for each integer $r \geq 2$ there exists an indecomposable rank $r$ vector bundle $B$ on $D$ with a filtration as above. In the set-up of doubly ruled three-folds $q$ is the genus of the base curve $C$.

\section{Further Examples of Bundles and Sheaves on Threefolds}

In our general set-up we have $X=\mathbb{P}(A)$ where $A$ is a rank two bundle on a surface $S$. Now we consider special cases where we have more concrete information.

Example 16. Fix an ample line bundle $L$ on $S$ and set $X^{\prime}:=\mathbb{P}\left(\mathcal{O}_{X} \oplus L\right)$. Let $v^{\prime}: X^{\prime} \rightarrow S$ denote the ruling. The projection $\pi_{1}: \mathcal{O}_{S} \oplus L \rightarrow \mathcal{O}_{S}$ induces a section of $v^{\prime}$ whose image $D^{\prime}$ has normal bundle $\pi^{*}(L) \mid S_{1}$. Hence $D$ has ample normal bundle and $X \backslash D \cong \operatorname{Tot}(L)$. However $D^{\prime}$ is not an ample divisor of $X^{\prime}$.

The projection $\mathcal{O}_{S} \oplus L \rightarrow L$ induces another section $D^{\prime \prime}$ of $v^{\prime}$ whose normal bundle $\pi_{2}: \mathcal{O}_{D^{\prime \prime}}\left(D^{\prime \prime}\right)$ is isomorphic to $L^{*}$ (up to the identification of $D^{\prime \prime}$ with $S$ given by the ruling $\left.v^{\prime}\right)$. Hence the conormal bundle of $D^{\prime \prime}$ in $X^{\prime}$ is ample. Since the kernels of the projections $\pi_{1}$ and $\pi_{2}$ are complementary, $D^{\prime} \cap D^{\prime \prime}=\emptyset$. We may see $D^{\prime \prime}$ as the obstruction to the ampleness of $D^{\prime}$ in $X^{\prime}$. For all integers $r>0, c_{1}, \ldots, c_{r}$, set

$$
A\left\{c_{1}, \ldots, c_{r}\right\}:=\oplus_{i=1}^{r} \mathcal{O}_{X^{\prime}}\left(c_{i} D^{\prime \prime}\right) .
$$

Since $D^{\prime \prime} \cap D^{\prime}=\emptyset$, we have $\left.A\left\{c_{1}, \ldots, c_{r}\right\}\right|_{D^{\prime}} \cong \mathcal{O}_{D^{\prime}}^{\oplus r}$. Restricting to each $D^{\prime \prime}$ we see that $A\left\{c_{1}, \ldots, c_{r}\right\} \cong A\left\{d_{1}, \ldots, d_{r}\right\}$ if and only if there is a permutation $\sigma:\{1, \ldots, r\} \rightarrow\{1, \ldots, r\}$ such that $d_{i}=c_{\sigma(i)}$ for all $i$. Obviously, $h^{0}\left(X^{\prime}, \mathcal{O}_{X^{\prime}}\left(c D^{\prime \prime}\right)\right)=0$ if $c<0$. Since $L$ is ample, we see that $D^{\prime \prime}$ appears with multiplicity $c$ in the base locus of the linear system $\left|c D^{\prime \prime}\right|$ on $X^{\prime}$. Hence

$$
h^{0}\left(X, A\left\{c_{1}, \ldots, c_{r}\right\}\right)=\#\left\{i \in\{1, \ldots, r\} ; c_{i} \geq 0\right\} .
$$

Example 17. Let $\alpha: U \rightarrow V$ be a $\mathbb{P}^{1}$-bundle. Hence every scheme-theoretic geometric fiber $F$ of $\alpha$ is isomorphic to $\mathbb{P}^{1}$. Thus $h^{0}\left(F, \mathcal{O}_{F}\right)=1$ and $h^{i}\left(F, \mathcal{O}_{F}\right)=$ 0 for all $i \geq 1$. The first equality gives $\alpha_{*}\left(\mathcal{O}_{U}\right)=\mathcal{O}_{V}$. Since $h^{i}\left(F, \mathcal{O}_{F}\right)=0$ for all $i \geq 1$, a base-change theorem ([7], p. 11) gives $R^{i} \alpha_{*}\left(\mathcal{O}_{U}\right)=0$ for all $i \geq 1$. Now assume that $V$ is complete. Hence $U$ is complete. The Leray spectral sequence of $\alpha$ gives $h^{i}\left(U, \mathcal{O}_{U}\right)=h^{i}\left(V, \mathcal{O}_{V}\right)$ for all $i \geq 0$. Applying the observations to the rulings $X \rightarrow D$ and $X^{\prime} \rightarrow D^{\prime}$ we get

$$
h^{1}\left(X, \mathcal{O}_{X}\right)=h^{1}\left(X^{\prime}, \mathcal{O}_{X^{\prime}}\right)=q
$$

and

$$
h^{i}\left(X, \mathcal{O}_{X}\right)=h^{i}\left(X^{\prime}, \mathcal{O}_{X^{\prime}}\right)=0
$$

for all $i \geq 2$. 
Proposition 18. With $D^{\prime}$ and $D^{\prime \prime}$ as in Example 16 we have

- $h^{1}\left(X, \mathcal{O}_{X^{\prime}}\left(-D^{\prime}\right)\right)=h^{1}\left(X, \mathcal{O}_{X^{\prime}}\left(-D^{\prime \prime}\right)\right)=0$.

- $h^{1}\left(X^{\prime}, \mathcal{O}_{X^{\prime}}\left(x D^{\prime \prime}-D^{\prime}\right)\right)=0$ for all integers $x \geq 0$.

- Fix an integer $y>0$, then

$$
h^{1}\left(X^{\prime}, \mathcal{O}_{X^{\prime}}\left(-y D^{\prime \prime}-D^{\prime}\right)\right) \leq \sum_{i=1} y h^{0}\left(S, L^{\otimes i}\right) .
$$

- If $h^{1}\left(S, L^{\otimes i}\right)=0$ for all $1 \leq i \leq y$, then

$$
h^{1}\left(X^{\prime}, \mathcal{O}_{X^{\prime}}\left(-y D^{\prime \prime}-D^{\prime}\right)\right)=\sum_{i=1} y h^{0}\left(S, L^{\otimes i}\right) .
$$

Proof: Consider the exact sequences on $X^{\prime}$

$$
\begin{gathered}
0 \rightarrow \mathcal{O}_{X^{\prime}}\left(c D^{\prime \prime}-D^{\prime}\right) \rightarrow \mathcal{O}_{X^{\prime}}\left(c D^{\prime \prime}-D^{\prime}\right) \rightarrow \mathcal{O}_{D^{\prime}}\left(c D^{\prime \prime}\right) \rightarrow 0 \\
0 \rightarrow \mathcal{O}_{X^{\prime}}\left((x-1) D^{\prime \prime}-D^{\prime}\right) \rightarrow \mathcal{O}_{X^{\prime}}\left(x D^{\prime \prime}-D^{\prime}\right) \rightarrow \mathcal{O}_{D^{\prime \prime}}\left(x D^{\prime \prime}-D^{\prime}\right) \rightarrow 0 .
\end{gathered}
$$

Recall that $\mathcal{O}_{D^{\prime}}\left(c D^{\prime \prime}\right) \cong \mathcal{O}_{D^{\prime}}$ for any $c$ because $D^{\prime} \cap D^{\prime \prime}=\emptyset$, also $\mathcal{O}_{D^{\prime \prime}}\left(x D^{\prime \prime}\right) \cong$ $L^{\otimes-x}$. Hence $\mathcal{O}_{D^{\prime \prime}}\left(x D^{\prime \prime}-D^{\prime}\right) \cong L^{\otimes-x}$. First take $c=0$ in sequence (9), then the natural map $H^{1}\left(X, \mathcal{O}_{D^{\prime}}\right) \rightarrow H^{1}\left(D, \mathcal{O}_{D^{\prime}}\right)$ is an isomorphism (use that $X^{\prime} \rightarrow D^{\prime}$ is a $\mathbb{P}^{1}$-bundle and the proof that $h^{1}\left(X, \mathcal{O}_{X^{\prime}}\right)=q$ given in Example 17). The restriction map $H^{0}\left(X, \mathcal{O}_{X^{\prime}}\right) \rightarrow H^{0}\left(X, \mathcal{O}_{X^{\prime}}\right)$ is obviously bijective. Hence $h^{1}\left(X, \mathcal{O}_{X}(-D)\right)=0$. In the same way using that $X^{\prime} \rightarrow D^{\prime \prime}$ is a $\mathbb{P}^{1}$-bundle we get $h^{1}\left(X, \mathcal{O}_{X^{\prime}}\left(-D^{\prime \prime}\right)\right)=0$.

Now we check that $h^{1}\left(X^{\prime}, \mathcal{O}_{X^{\prime}}\left(x D^{\prime \prime}-D^{\prime}\right)\right)=0$ by induction on $x$. We proved that this vanishing is true if $x=0$. Assume $x>0$ and that this vanishing is true for the integer $x^{\prime}:=x-1$. Since $L$ is ample on $D^{\prime \prime}$ and $x>0$, Kodaira vanishing gives $h^{1}\left(X, L^{\otimes-x}\right)=0$. Thus, sequence (10) gives $h^{1}\left(X^{\prime}, \mathcal{O}_{X^{\prime}}\left(x D^{\prime \prime}-D^{\prime}\right)\right)=0$.

We check the last two assertions by induction on $y$, starting from the case $y=0$ which is true, because $h^{1}\left(X, \mathcal{O}_{X^{\prime}}\left(-D^{\prime}\right)\right)=0$. Assume that the last assertions are true for the integer $y^{\prime}:=y-1$. Then use the sequence (10) with $x:=y-1$ to prove both assertions.

Proposition 19. $h^{2}\left(S, L^{\otimes k}\right)=0$ for all $k \geq 0$.

Proof: Let $u: S \rightarrow C$ be the ruling of $S$. By Serre duality it is sufficient to prove $h^{0}\left(S, L^{\otimes-k} \otimes \omega_{S}\right)=0$, i.e., that $h^{0}\left(C, u_{*}\left(L^{\otimes-k} \otimes \omega_{S}\right)\right)=0$. Let $T \cong \mathbb{P}^{1}$ be a fiber of $u$. Since $L$ is ample, $L \mid T$ has degree $>0$. Since $\omega_{S}$ has degree -2 and $k \geq 0, L^{\otimes-k} \otimes \omega_{S} \mid T$ has degree $<0$. Hence $u_{*}\left(L^{\otimes-k} \otimes \omega_{S}\right)=0$ and it follows that $h^{0}\left(C, u_{*}\left(L^{\otimes-k} \otimes \omega_{S}\right)\right)=0$. 
Example 20. Fix an integer $k>0$ and assume $h^{1}\left(X, L^{\otimes k}\right)=0$. Proposition 19 gives $h^{2}\left(S, L^{\otimes k}\right)=0$. By Riemann-Roch $h^{0}\left(S, L^{\otimes k}\right)=k^{2} L^{2}-k \omega_{S} \cdot L+1-q$. Consider the following property that $L$ may have

$$
\text { property }(+) h^{1}\left(S, L^{\otimes i}\right)=0 \text { for all integers } i>0 .
$$

If $L$ has Property (+), then Proposition 18 shows that the integer

$$
h^{1}\left(X, \operatorname{Hom}\left(A_{c_{1}, \ldots, c_{r}}, A_{c_{1}, \ldots, c_{r}}\right)\left(-D^{\prime}\right)\right)
$$

is uniquely determined by the integers $r, c_{1}, \ldots, c_{r}$ and the intersection numbers $L^{2}$ and $\omega_{S} \cdot L$. Recall that $H^{1}\left(X, \operatorname{Hom}\left(A\left\{c_{1}, \ldots, c_{r}\right\}, A\left\{c_{1}, \ldots, c_{r}\right\}\right)\left(-D^{\prime}\right)\right)$ is the tangent space at $A\left\{c_{1}, \ldots, c_{r}\right\}$ of the deformation functor of $A\left\{c_{1}, \ldots, c_{r}\right\}$ with fixed restriction to $D^{\prime}$.

Fix any ample line bundle $G$ on $S$. Since $G$ is ample, there is an integer $k_{G}>0$ such that $h^{1}\left(S, G^{\otimes k}\right)=0$ for all integers $k \geq k_{G}$. Hence for any integer $k \geq k_{G}$ the ample line bundle $L:=G^{\otimes k}$ has property (+). Thus, in all these cases the tangent space to the deformation functor is entirely determined by the numerical data $r, c_{1}, \cdots, c_{r}, L^{2}$, and $\omega_{S} \cdot L$.

\section{Acknowledgements}

This article was completed during a RIGs program supported by the Fondazione Bruno Kessler at CIRM Trento, we are grateful for the support and wonderful hospitality of that institute. The first author was partially supported by MIUR and GNSAGA of INdAM.

\section{References}

[1] Eisenbud D., Commutative Algebra with a View Toward Algebraic Geometry, Springer, Berlin, 1995.

[2] Félix Y., Oprea J. and Tanré D., Algebraic Models in Geometry, Oxford Univ. Press, Oxford, 2008.

[3] Gasparim E., Köppe T. and Majumdar P., Local Holomorphic Euler Characteristic and Instanton Decay, Pure Appl. Math. Q. 4 (2008) 161-179.

[4] Hartshorne R., Ample Subvarieties of Algebraic Varieties, LNM vol. 156, Springer, Berlin, 1970. 
[5] Hartshorne R., Stable Reflexive Sheaves, Math. Ann. 254 (1980) 121-176.

[6] Lübke M. and Teleman A., The Kobayashi-Hitchin Correspondence, World Scientific, River Edge, 1997.

[7] Okonek C., Schneider M. and Spindler H., Vector Bundles on Projective Spaces, Birkhäuser, Boston, 1980.

Edoardo Ballico

Department of Mathematics

University of Trento

38050 Povo (TN), ITALY

E-mail address: ballico@science.unitn. it

Elizabeth Gasparim

School of Mathematics

University of Edinburgh

The King's Buildings

JCMB, Mayfield Road

EH9 3JZ Edinburgh, SCOTLAND

E-mail address: Elizabeth. Gasparimeed.ac.uk 